\title{
Does ventral mesh rectopexy at the time of sacrocolpopexy prevent subsequent posterior wall prolapse?
}

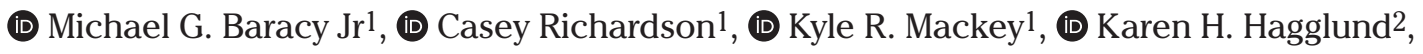 \\ (D) Muhammad Faisal Aslam 3,4 \\ 1Department of Obstetrics and Gynecology, Ascension St. John Hospital, Michigan, United States of America \\ 2Department Biomedical Investigations and Research, Ascension St. John Hospital, Michigan, United States of America \\ 3Department of Female Pelvic Medicine and Reconstructive Surgery, Ascension St. John Hospital, Michigan, \\ United States of America \\ 4Department of Female Pelvic Medicine and Reconstructive Surgery, Michigan State University, Michigan, \\ United States of America
}

\section{Abstract}

Objective: To determine whether ventral mesh rectopexy at the time of sacrocolpopexy reduces the rate of future posterior wall prolapse.

Material and Methods: This was a retrospective cohort study of women with pelvic organ prolapse (POP) who underwent sacrocolpopexy or without concomitant rectopexy at a single community hospital from December 1, 2015 to June 30, 2019. Preoperative pelvic organ prolapse quantification (POP-Q) and urodynamic testing was used in evaluation of POP. Patients were followed for 12-weeks postoperatively and a 12week postoperative POP-Q assessment was completed. The incidence of new or recurrent posterior prolapse was compared between cohorts.

Results: Women with POP $(n=150)$ were recruited, of whom $41(27.3 \%)$ underwent sacrocolpopexy while the remainder $(n=109,72.7 \%)$ did not receive rectopexy. Patient demographics did not statistically differ between cohorts. Post-surgical posterior wall prolapse was reduced in the robotic assisted sacrocolpopexy (RASC) + rectopexy group compared to RASC alone, however this did not reach statistical significance. There were no patients who underwent concomitant rectopexy and RASC that needed recurrent posterior wall prolapse surgery, compared to eightpercent of patients that underwent isolated RASC procedures.

Conclusion: Our findings suggest a reduction in the need for subsequent posterior wall surgery when rectopexy is performed at the time of sacrocolpopexy. In our study, no future surgery for POP was found in the concomitant sacrocolpopexy and rectopexy group, while a small proportion of the RASC only group required future POP surgery. Our study, however, was underpowered to elucidate a statistically significant difference between groups. Future larger studies are needed to confirm a reduced risk of posterior wall prolapse in patients who undergo concomitant RASC and rectopexy. (J Turk Ger Gynecol Assoc 2021; 22: 174-80)

Keywords: Pelvic organ prolapse, sacrocolpopexy, rectopexy, minimally invasive surgery, multicompartmental prolapse

Received: 14 February, 2021 Accepted: 06 June, 2021

\section{Introduction}

Pelvic floor disorders are a common and costly healthcare concern. It is estimated that one in four adult women in the United States experience pelvic floor dysfunction, with prevalence increasing with age (1). Pelvic organ prolapse (POP) occurs when the muscles that hold the pelvic organs (e.g. uterus, bladder, and or rectum) weaken and these organs are displaced from their normal anatomical position, typically resulting in protrusion of the anterior or posterior vaginal wall

Address for Correspondence: Michael G. Baracy Jr 
into the vagina. By the age of 60 years, more than one-third of women will have one or more pelvic floor disorders (2). The lifetime risk of undergoing pelvic floor surgery is estimated to be $11 \%-20 \%(3,4)$. Furthermore, nearly $30 \%$ of patients who undergo surgical correction of POP will require reoperation for recurrent prolapse or incontinence (3).

There are several treatment modalities for POP, including expectant management, conservative approaches, and surgical correction. Conservative measures include pessary and pelvic floor physical therapy, which has been shown to be effective for early stages of POP (5). In patients with more severe POP, which may be assessed with tools such as the preoperative pelvic organ prolapse quantification (POP-Q) with more severe POP being indicated with a staging of POP-Q 3 or 4 , pessaries and surgical options have been shown to have similar outcomes $(6,7)$. Surgical options can be obliterative or reconstructive in nature. Obliterative surgery corrects prolapse by removing portions of the vagina, narrowing part of the vagina, or closing off the vagina entirely (8). Conversely, reconstructive surgery aims to restore normal anatomy through either the use of natural structures, such as ligaments, or by creating pelvic organ support with interposition of synthetic mesh (9). Of the surgical options, minimally invasive sacrocolpopexy has become the "gold standard" in POP repair (10-13).

When compared to vaginal procedures for POP, sacrocolpopexy has been shown to be more efficacious, resulting in decreased risk of recurrent prolapse and the need for repeat surgery, decreased risk of postoperative stress urinary incontinence, and decreased risk of sexual side effects (e.g. dyspareunia) compared to vaginal approaches $(12,13)$. Furthermore, studies have shown that apical support reduces anterior vaginal wall prolapse (14-16), owing to the effectiveness of sacrocolpopexy. As a result, minimally invasive sacrocolpopexy has become the treatment of choice for advanced stage POP. Unfortunately, even after repair with sacrocolpopexy, recurrence of $\mathrm{POP}$ has been reported in up to $23.2 \%$ of patients, with risk increasing with greater presurgical clinical severity and stage (17).

Multicompartmental prolapse complicates the treatment of POP. It is estimated that $10-55 \%$ of patients with POP have two or more concomitant pelvic floor disorders $(18,19)$. The organs involved can include any combination of the bladder and anterior vagina, the posterior vagina and rectum, the uterus, the vaginal cuff in patients status post hysterectomy, the perineum, and partial- or full-thickness rectum. Traditionally, rectal prolapse and vaginal prolapse have been regarded as separate entities and treated by disparate surgical procedures $(20,21)$. Minimally invasive ventral mesh rectopexy has become increasingly used in the treatment of posterior compartment defects (22-24). Recently, there has been a shift from a compartmentalized approach toward concomitant procedures for correction of multicompartmental prolapse. Accordingly, ventral mesh rectopexy at the time of sacrocolpopexy is increasingly performed to treat rectal prolapse $(20,21)$. Currently, however, there is a paucity of research examining the effects of rectopexy at the time of sacrocolpopexy and subsequent posterior vaginal wall prolapse.

To date, no study has investigated ventral mesh rectopexy at the time of sacrocolpopexy for the prevention of future posterior vaginal wall prolapse. The aim of this study was to determine whether ventral mesh rectopexy, completed at the time of sacrocolpopexy for apical vaginal prolapse, can reduce future posterior wall prolapse recurrence.

\section{Material and Methods}

This is a retrospective cohort study of women aged 18-years or older who underwent minimally invasive roboticassisted sacrocolpopexy (RASC) at an urban community teaching hospital from December 1, 2015 to June 30, 2019 (ICD 10 CPT 57425). Patients were stratified depending on if they underwent ventral mesh rectopexy at the time of sacrocolpopexy or if they only had a sacrocolpopexy procedure. Each patient had medical clearance by a primary medical provider prior to surgery. Each patient underwent a preoperative presurgical POP-Q interactive assessment (25). Each POP-Q was performed by one board-certified female pelvic medicine and reconstructive surgeon.

Urodynamic testing was performed for each patient to determine the presence of occult stress incontinence or detrusor overactivity. If the patient was determined to have occult stress urinary incontinence, a tension-free vaginal tape (TVT) was performed at the time of the procedure. Patients with posterior wall prolapse on POP-Q or fecal incontinence underwent magnetic resonance (MR) defecography. Based on the results of the POP-Q and MR defecography or if the patient had rectal prolapse (internal, partial, complete) they underwent ventral wall rectopexy at the time of RASC. Each ventral mesh rectopexy was performed by a single, board-certified, colorectal surgeon. If the patient had not previously undergone a hysterectomy, a robotic assisted total hysterectomy was performed. A Y-shaped lightweight polypropylene mesh (Vertessa $^{\circledast}$ Lite, Caldara Medical, Augora Hills, CA, USA) was used for the sacrocolpopexy. A 0- delayed-absorbable polydioxanone suture (PDS) in a running fashion was used for the anterior and posterior arms of the vaginal mesh. The vaginal cuff (where applicable) was closed with 0-PDS suture in a running fashion. The mesh was affixed to the sacral promontory with a 0-braided polyester (TiCron, Covidien, Minneapolis, MN, USA), nonabsorbable suture via two simple interrupted sutures. 
Patient demographics, medical comorbidities, past medical history, surgical history, obstetric history and social habits were recorded for each patient undergoing POP repair. Operative complications, postoperative complications, and hospital length of stay were analyzed. The study was approved and deemed exempt by the Ascension St. John Institutional Review Board (approval number: \#1477635). However, because this was a retrospective study, informed consent was not obtained. Success of rectopexy with concomitant RASC was determined by the absence of posterior vaginal wall prolapse in subsequent postoperative visits. Patients were followed for a total of 12 weeks postoperatively. A POP-Q assessment was performed at each patient's 12-week postoperative evaluation. Failure was defined as posterior vaginal wall prolapse recurrence using the POP-Q interactive assessment tool, defined as Ap or Bp greater than point 0 - beyond the hymenal ring, or the need for future surgery for posterior vaginal wall prolapse. Point $\mathrm{C}$ was considered only in the context of Ap or Bp, owing to the fact that for deviation of point $\mathrm{C}$, Bp would be expected to be affected in posterior wall prolapse.

\section{Statistical analysis}

Data were tested for normality and homogeneity of variance. Normally distributed data was reported as mean \pm standard deviation. Non-normal data was reported as median + interquartile range. A paired t-test was used to compare preoperatively and postoperative POP-Q values. Univariate analyses were conducted with Student's t-test, the chi-squared test, and analysis of variance (ANOVA). Multivariate analyses were done using logistic regression. A p-value $<0.05$ was considered statistically significant. Statistical analysis was performed using SPSS Software v. 25.0 (IBM, Armonk, NY, USA). There is a paucity of literature examining prevention of posterior wall prolapse recurrence by performing rectopexy at time of sacrocolpopexy. Accordingly, our power analysis was limited to estimations based on observations at our institution. For our power analysis, we estimated that the posterior wall recurrence without rectopexy was approximately $25 \%$ and two-percent with concomitant rectopexy. Using this criterion, it was estimated that 141 subjects in each group would be necessary to detect a difference at a power of $80 \%$ and $\alpha=0.05$.

\section{Results}

In total, 150 women were retrospectively reviewed. Of these 41 (27.3\%) underwent RASC with concomitant rectopexy while the remaining 109 (72.7\%) underwent only RASC (Figure 1). Baseline demographic variables, including race, body mass index, parity, diabetes mellitus and smoking were not statistically different between groups (Table 1). Patients undergoing RASC alone had greater proportion of advanced stage POP compared to patients undergoing RASC + Rectopexy, (76\% stage 3, 23\% stage 4 vs 68\% stage 3, 5\% stage 4, p<0.0001) (Table 2). Furthermore, preoperative POP-Q scores were significantly different for points Aa (1.3 \pm 1.6 vs $2.7 \pm 1.0, \mathrm{p}<0.0001)$, $\mathrm{Ba}(1.8 \pm 2.2$ vs $4.5 \pm 2.3, \mathrm{p}<0.0001)$, $\mathrm{C}(-2.1 \pm 4.5$ vs $2.6 \pm 4.6, \mathrm{p}<0.0001), \mathrm{Pb}(3.1 \pm 1.0$ vs $2.5 \pm 1.0$, $\mathrm{p}=0.002)$, Ap $(0.0 \pm 1.8$ vs $-1.2 \pm 1.7 \mathrm{~cm}, \mathrm{p}=0.001)$, and $\mathrm{Bp}$ $(0.3 \pm 2.2$ vs $-1.2 \pm 1.4 \mathrm{~cm}, \mathrm{p}<0.0001)$ for patients undergoing RASC and rectopexy comparted with RASC alone. Patients undergoing RASC alone had greater anterior prolapse but less posterior compartment prolapse compared to patients who underwent concomitant RASC and rectopexy (Table 2). Concurrent surgeries were similar between groups with the exception of bilateral-salpingoophorectomy, which was completed more often in the RASC group, $(p=0.014$; Table 3).

Overall there was a low complication rate. Within the sacrocolpopexy alone cohort there was one umbilical port hernia, one rectal injury, and two cystotomies at the time of TVT placement. In the RASC and rectopexy group, there was one mesenteric bleed with hemoperitoneum that was controlled with a hemostatic agent (Table 4). Congruent with these results, hospital length of stay (in days) did not statistically differ between RASC and RASC + rectopexy $(1.1 \pm 0.5$ vs $1.3 \pm 0.7$, $\mathrm{p}=0.071$ ). In the combined cohort, postoperative POP-Q scores were significantly improved for $\mathrm{Aa}, \mathrm{Ab}, \mathrm{C}, \mathrm{Gh}, \mathrm{Ap}$, and $\mathrm{Bp}$ ( $<<0.0001$ for each) (Supplemental Table 1). When comparing postoperative POP-Q scores, point $\mathrm{C}$ was the only score that significantly differed, with RASC alone having slightly higher point C ( $-9.3 \pm 1.5 \mathrm{~cm}$ vs $-8.4 \pm 3.3 \mathrm{~cm}, \mathrm{p}=0.036)$ (Table 5).

Recurrence and/or subsequent postsurgical posterior compartment prolapse were found in $10 \%$ of the RASC and $3 \%$ of the RASC + Rectopexy cohort $(\mathrm{p}=0.181)$. Eight-percent of patients in the RASC cohort had to undergo subsequent posterior repair, while no patients in the RASC + rectopexy cohort needed repeat surgery $(p=0.114)$ (Table 6).

\section{Discussion}

There is a paucity of research examining the recurrence or subsequent posterior wall prolapse following concomitant sacrocolpopexy and rectopexy compared to sacrocolpopexy alone. The aim of this study was to determine if concomitant ventral mesh rectopexy during minimally-invasive RASC reduced the rate of subsequent posterior vaginal wall prolapse. To our knowledge, this is the first study to investigate this matter. The Colpopexy and Urinary Reduction Effort trial did investigate the impact of POP surgery on posterior compartment symptoms; however, posterior colporraphy rather than ventral mesh rectopexy was performed at the discretion of the surgeon 


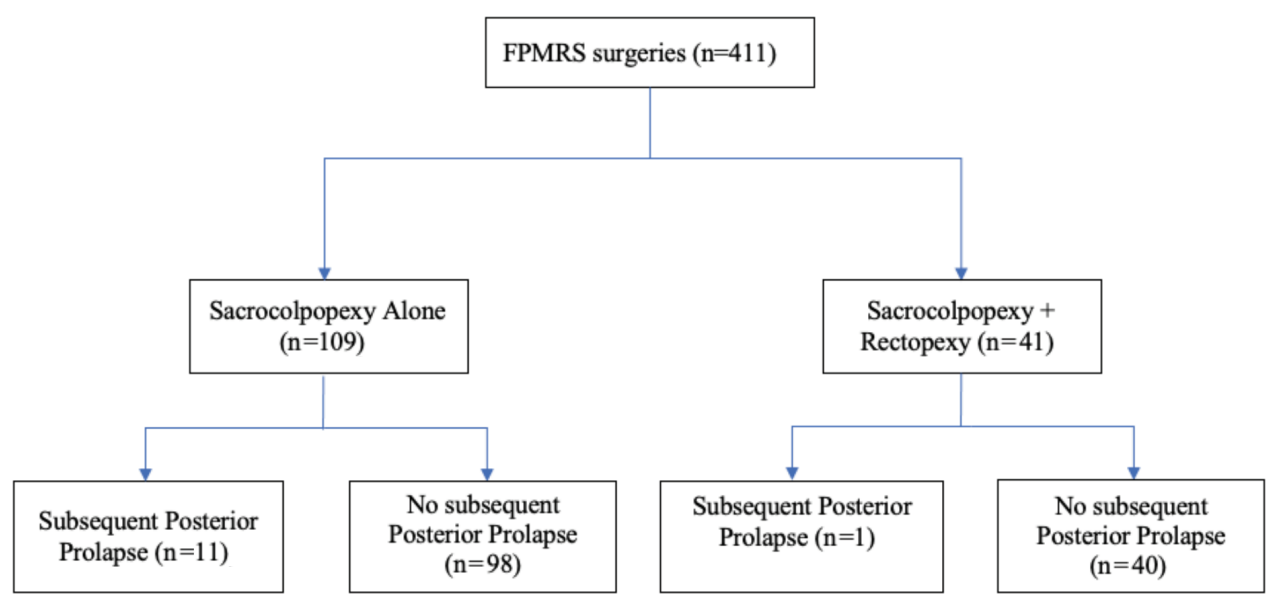

Figure 1. Study cohorts

FPMRS: Female Pelvic Medicine and Reconstructive Surgery

Table 1. Patient demographics and medical comorbidities

\begin{tabular}{|l|l|l|l|}
\hline \multirow{2}{*}{ Characteristic } & RASC & $\begin{array}{l}\text { RASC + } \\
\text { Rectopexy }\end{array}$ & \multirow{2}{*}{ p } \\
\cline { 2 - 3 } & $\mathbf{n = 1 0 9}$ & $\mathbf{n = 4 1}$ & \\
\hline Age (years) & $63.3 \pm 8.4$ & $62.8 \pm 12.9$ & 0.792 \\
\hline Race & $88(82)$ & $33(83)$ & \multirow{2}{*}{1.00} \\
\hline White/Caucasian & $20(18)$ & $7(17)$ & \\
\hline Black/African American & $28.5 \pm 5.0$ & $27.5 \pm 4.8$ & 0.264 \\
\hline BMI & $2.9 \pm 1.4$ & $2.8 \pm 1.4$ & 0.693 \\
\hline Parity & $11(10)$ & $5(12)$ & 0.768 \\
\hline Diabetes mellitus & $8(7)$ & $7(17)$ & 0.122 \\
\hline Smoking & $\begin{array}{l}\mid l \\
\text { All data reported as mean } \pm \text { standard deviation or n (\%). }\end{array}$ \\
$\begin{array}{l}\text { RASC: Robotic Assisted Laparoscopic Sacrocolpopexy, BMI: Body mass } \\
\text { index in kg/m² }\end{array}$ & \\
\hline
\end{tabular}

(26). Ultimately, the authors found that the anorectal symptoms improved in both the posterior colporraphy group and those without posterior repair at the time of POP surgery (26).

In our study, preoperative and postoperative POP-Q measurements predictably improved following surgical intervention, regardless of cohort. Our findings are consistent with a meta-analysis by Hudson et al. (27), who observed an overall anatomical success rate of $98.6 \%$ (27). However, the mean follow-up from this meta-analysis was $26.9 \pm 17.3$ months as compared to our study that used a relatively short 12 -week follow-up period.

Patients in the RASC alone group had greater anterior prolapse and significantly greater stage of prolapse compared to RASC and rectopexy (Table 2). This could have contributed to the increased risk of future posterior wall prolapse found in the RASC group. However, we do not believe that this would entirely account for our findings. Patients in the concomitant
Table 2. Preoperative pelvic organ prolapse quantification and prolapse stage

\begin{tabular}{|c|c|c|c|}
\hline \multirow{2}{*}{ POP-Q } & RASC & $\begin{array}{l}\text { RASC + } \\
\text { Rectopexy }\end{array}$ & \multirow{2}{*}{$\mathbf{p}$} \\
\hline & $n=109$ & $n=41$ & \\
\hline Aa & $2.7 \pm 1.0$ & $1.3 \pm 1.6$ & $<0.0001$ \\
\hline $\mathrm{Ba}$ & $4.5 \pm 2.3$ & $1.8 \pm 2.2$ & $<0.0001$ \\
\hline $\mathrm{C}$ & $2.6 \pm 4.6$ & $-2.1 \pm 4.5$ & $<0.0001$ \\
\hline Gh & $5.1 \pm 1.5$ & $4.7 \pm 1.4$ & 0.197 \\
\hline $\mathrm{Pb}$ & $2.5 \pm 1.0$ & $3.1 \pm 1.0$ & 0.002 \\
\hline TVL & $9.6 \pm 1.4$ & $9.1 \pm 1.9$ & 0.113 \\
\hline Ap & $-1.2 \pm 1.7$ & $0.0 \pm 1.8$ & 0.001 \\
\hline Bp & $-1.2 \pm 1.4$ & $0.3 \pm 2.2$ & $<0.0001$ \\
\hline $\mathrm{D}$ & $-4.6 \pm 2.3$ & $-5.3 \pm 1.1$ & 0.236 \\
\hline \multicolumn{4}{|l|}{ Stage } \\
\hline Stage 2 & $1(1)$ & $11(27)$ & \multirow{3}{*}{$<0.0001$} \\
\hline Stage 3 & $83(76)$ & $28(68)$ & \\
\hline Stage 4 & $25(23)$ & $2(5)$ & \\
\hline
\end{tabular}

Table 3. Concomitant surgeries at time of sacrocolpopexy

\begin{tabular}{|l|l|l|l|}
\hline \multirow{2}{*}{ Surgery } & RASC & RASC + Rectopexy & \multirow{2}{*}{$\mathbf{p}$} \\
\cline { 2 - 4 } & $\mathbf{n = 1 0 9}$ & $\mathbf{n = 4 1}$ & 0.098 \\
\hline Hysterectomy & $55(51)$ & $14(34)$ & 1.000 \\
\hline Bilateral salpingectomy & $22(20)$ & $8(20)$ & $\mathbf{0 . 0 1 4}$ \\
\hline BSO & $35(32)$ & $5(12)$ & 0.819 \\
\hline Tension free vaginal tape & $88(81)$ & $34(83)$ & Bilateral \\
\hline $\begin{array}{l}\text { All data reported as mean } \pm \text { standard deviation or n (\%). } \\
\text { RASC: Robotic Assisted Laparoscopic Sacrocolpopexy, BSO: } \\
\text { salphigo-oopherectomy }\end{array}$ &
\end{tabular}


RASC and Rectopexy cohort, however, had significantly greater posterior compartment defects as indicated by their POP-Q assessment. These findings are consistent with anatomical defects which would lead to the necessity of posterior repair. Multicompartmental prolapse is evident in approximately $10-55 \%$ of patients with POP $(18,19)$. Recently, there has been a shift from disparate, compartmentalized procedures toward concomitant procedures for correction of multicompartmental prolapse. Increasingly, ventral mesh rectopexy is being performed at the time of sacrocolpopexy for rectal prolapse $(20,21)$. Our study evaluated ventral mesh rectopexy at the time of sacrocolpopexy and subsequent incidence of posterior wall vaginal prolapse. Our findings show a trend in reducing subsequent posterior wall prolapse when ventral mesh rectopexy was performed at the time of sacrocolpopexy. Patients who underwent ventral mesh rectopexy at the time of RASC showed better posterior wall integrity on the POP-Q assessment and also required less posterior wall corrective surgery compared to patients who underwent sacrocolpopexy alone. This reduction in subsequent posterior prolapse, however, was not statistically significant, secondary to inadequate sample size. Our study lacked power to discern a statistically significant difference

Table 4. Operative/postoperative complications and length of hospital stay

\begin{tabular}{|c|c|c|c|}
\hline \multirow[t]{2}{*}{ Complication } & RASC & $\begin{array}{l}\text { RASC + } \\
\text { Rectopexy }\end{array}$ & \multirow{2}{*}{$\mathbf{p}$} \\
\hline & $n=109$ & $n=41$ & \\
\hline Bowel/mesenteric injury & $1(0.9)$ & $1(2.4)$ & 0.473 \\
\hline Port site hernia & $1(0.9)$ & $0(0)$ & 1.000 \\
\hline Length of hospital stay (days) & $1.1 \pm 0.5$ & $1.3 \pm 0.7$ & 0.071 \\
\hline
\end{tabular}

\section{Supplemental Table 1. Pre/postoperative POP-Q} values for combined cohorts

\begin{tabular}{|l|l|l|l|}
\hline POP-Q & Preoperative & Postoperative & p \\
\hline $\mathrm{Aa}$ & $2.3 \pm 1.3$ & $-2.7 \pm 0.5$ & $<\mathbf{0 . 0 0 0 1}$ \\
\hline $\mathrm{Ba}$ & $3.7 \pm 2.6$ & $-2.7 \pm 0.6$ & $<\mathbf{0 . 0 0 0 1}$ \\
\hline $\mathrm{C}$ & $1.4 \pm 4.9$ & $-9.0 \pm 2.1$ & $<\mathbf{0 . 0 0 0 1}$ \\
\hline $\mathrm{Gh}$ & $5.0 \pm 1.5$ & $3.4 \pm 2.2$ & $<\mathbf{0 . 0 0 0 1}$ \\
\hline $\mathrm{Pb}$ & $2.7 \pm 1.0$ & $2.7 \pm 0.7$ & 0.775 \\
\hline $\mathrm{TVL}$ & $9.4 \pm 1.6$ & $9.5 \pm 1.1$ & 0.725 \\
\hline $\mathrm{Ap}$ & $-0.8 \pm 1.8$ & $-2.2 \pm 0.8$ & $<\mathbf{0 . 0 0 0 1}$ \\
\hline $\mathrm{Bp}$ & $-0.8 \pm 1.8$ & $-2.2 \pm 0.8$ & $<\mathbf{0 . 0 0 0 1}$ \\
\hline $\mathrm{Data}$ & & &
\end{tabular}

Data reported as mean \pm standard deviation.

POP-Q: Postoperative pelvic organ prolapse quantification, TVL: Total Vaginal Length when in reference to POP-Q values and an appropriately powered study is required to confirm or refute this trend.

\section{Study limitation}

There are several limitations of this study. The retrospective nature of the study and the convenience sample are inherently prone to selection bias. However, this study provides an initial framework on which future prospective studies can be based. Furthermore, this study did not control for all medical comorbidities or confounding factors. Additionally, this study was underpowered to elucidate a statistical significance. However, we are currently conducting a study that addresses these issues and has an adequate sample size. Finally, subsequent posterior wall prolapse defined by POP-Q postoperatively was limited to 12 weeks of postoperative follow-up. It is possible that there could be posterior compartment prolapse past the follow-up

Table 5. Postoperative pelvic organ prolapse quantification and stage prolapse stage

\begin{tabular}{|c|c|c|c|}
\hline \multirow[t]{2}{*}{ POP-Q } & RASC & $\begin{array}{l}\text { RASC + } \\
\text { Rectopexy }\end{array}$ & \multirow[t]{2}{*}{$\mathbf{p}$} \\
\hline & $n=109$ & $n=41$ & \\
\hline Aa & $-2.7 \pm 0.5$ & $-2.7 \pm 0.4$ & 0.706 \\
\hline $\mathrm{Ba}$ & $-2.7 \pm 0.6$ & $-2.7 \pm 0.4$ & 0.882 \\
\hline $\mathrm{C}$ & $-9.3 \pm 1.5$ & $-8.4 \pm 3.3$ & 0.036 \\
\hline Gh & $3.4 \pm 2.2$ & $3.3 \pm 2.3$ & 0.829 \\
\hline $\mathrm{Pb}$ & $2.6 \pm 0.7$ & $2.8 \pm 0.7$ & 0.226 \\
\hline TVL & $9.5 \pm 1.2$ & $9.3 \pm 1.0$ & 0.200 \\
\hline Ap & $-2.1 \pm 0.8$ & $-2.3 \pm 0.9$ & 0.496 \\
\hline $\mathrm{Bp}$ & $-2.2 \pm 0.7$ & $-2.3 \pm 0.9$ & 0.606 \\
\hline $\mathrm{D}$ & $\mathrm{n} / \mathrm{a}$ & $\mathrm{n} / \mathrm{a}$ & - \\
\hline Stage & $n=103$ & $n=40$ & - \\
\hline Stage 0 & $12(12)$ & $8(20)$ & \multirow{3}{*}{0.423} \\
\hline Stage 1 & $81(78)$ & $28(70)$ & \\
\hline Stage 2 & $10(10)$ & $4(10)$ & \\
\hline \multicolumn{4}{|c|}{$\begin{array}{l}\text { All data reported as mean } \pm \text { standard deviation or } n(\%) \text {. } \\
\text { RASC: Robotic Assisted Laparoscopic Sacrocolpopexy, POP-Q: } \\
\text { Postoperative pelvic organ prolapse quantification }\end{array}$} \\
\hline
\end{tabular}

Table 6. Outcomes and failure rates

\begin{tabular}{|l|l|l|l|}
\hline \multirow{2}{*}{ Outcome } & RASC & $\begin{array}{l}\text { RASC + } \\
\text { Rectopexy }\end{array}$ & \multirow{2}{*}{ p } \\
\cline { 2 - 4 } & $\mathbf{n = 1 0 9}$ & $\mathbf{n = 4 1}$ & \\
\hline Subsequent posterior repair & $9(8)$ & $0(0)$ & 0.114 \\
\hline Failure (POP-Q definition) & $11(10)$ & $1(3)$ & 0.181 \\
\hline Other reoperation & $3(3)$ & $4(10)$ & 0.088 \\
\hline $\begin{array}{l}\text { POP-Q: Postoperative Pelvic Organ Prolapse Quantification (POP-Q) } \\
\text { Failure (POP-Q) defined as Ap or Bp greater than point 0 (beyond the } \\
\text { hymenal ring). } \\
\text { RASC: Robotic Assisted Laparoscopic Sacrocolpopexy }\end{array}$ \\
\hline
\end{tabular}


period. However, we believe most failures would be evident in the three months postoperative timeframe.

Strengths of this study include its novelty. We believe that this is the first study to investigate the effect of concomitant rectopexy at the time of RASC and the recurrence of posterior wall prolapse. Furthermore, all the procedures were performed by one fellowship trained board certified surgeon in their respective fields, allowing consistency of technique and skill. In addition, all patients underwent standardized POP-Q evaluation both pre- and postoperatively.

Our study elucidated a trend towards fewer occurrences of posterior vaginal wall prolapse when ventral mesh rectopexy is performed at the time of sacrocolpopexy. Our study was underpowered, however, to prove a statistically significant difference.

\section{Conclusion}

It is possible that ventral mesh rectopexy provides important structural support of the posterior vaginal wall thereby reducing subsequent posterior prolapse. Overall there was a low complication rate, and surgical complications were not statistically different between groups. Additionally, hospital length of stay did not differ between groups. Patients at risk for posterior wall prolapse, for example POP-Q assessment demonstrating posterior wall defects, might benefit from concomitant ventral mesh rectopexy at the time of sacrocolpopexy for POP, without addition surgical complications or longer hospital stays. An adequately powered study is needed, however, to confirm these findings.

Ethics Committee Approval: The study was approved and deemed exempt by the Ascension St. John Institutional Review Board (approval number: \#1477635).

Informed Consent: This was a retrospective study, informed consent was not obtained.

Peer-review: Externally peer-reviewed.

Author Contributions: Surgical and Medical Practices: M.F.A.; Concept: M.F.A., C.R.; Design: M.F.A., C.R., K.H.H.; Data Collection or Processing: C.R.; Analysis or Interpretation: C.R., K.H.H.; Literature Search: K.R.M., M.G.B.J.; Writing: K.R.M., M.G.B.J.

Conflict of Interest: No conflict of interest is declared by the authors.

Financial Disclosure: The authors declared that this study received no financial support.

\section{References}

1. Wu JM, Vaughan CP, Goode PS, Redden DT, Burgio KL, Richter HE, et al. Prevalence and trends of symptomatic pelvic floor disorders in U.S. women. Obstet Gynecol 2014; 123: 141-8.

2. Wu JM, Matthews CA, Conover MM, Pate V, Jonsson Funk M. Lifetime risk of stress urinary incontinence or pelvic organ prolapse surgery. Obstet Gynecol 2014; 123: 1201-6.

3. Olsen AL, Smith VJ, Bergstrom JO, Colling JC, Clark AL. Epidemiology of surgically managed pelvic organ prolapse and urinary incontinence. Obstet Gynecol 1997; 89: 501-6.

4. Smith FJ, Holman CD, Moorin RE, Tsokos N. Lifetime risk of undergoing surgery for pelvic organ prolapse. Obstet Gynecol 2010; 116: 1096-100.

5. Li C, Gong Y, Wang B. The efficacy of pelvic floor muscle training for pelvic organ prolapse: a systematic review and meta-analysis. Int Urogynecol J 2016; 27: 981-92.

6. Lone F, Thakar R, Sultan AH. One-year prospective comparison of vaginal pessaries and surgery for pelvic organ prolapse using the validated ICIQ-VS and ICIQ-UI (SF) questionnaires. Int Urogynecol J 2015; 26: 1305-12.

7. Abdool Z, Thakar R, Sultan AH, Oliver RS. Prospective evaluation of outcome of vaginal pessaries versus surgery in women with symptomatic pelvic organ prolapse. Int Urogynecol J 2011; 22: 273-8.

8. Abbasy S, Kenton K. Obliterative procedures for pelvic organ prolapse. Clin Obstet Gynecol 2010; 53: 86-98.

9. Brubaker L, Maher C, Jacquetin B, Rajamaheswari N, von Theobald P, Norton P. Surgery for pelvic organ prolapse. Female Pelvic Med Reconstr Surg 2010; 16: 9-19.

10. Ganatra AM, Rozet F, Sanchez-Salas R, Barret E, Galiano $\mathrm{M}$, Cathelineau $\mathrm{X}$, et al. The current status of laparoscopic sacrocolpopexy: a review. Eur Urol 2009; 55: 1089-103.

11. Maher CF, Feiner B, DeCuyper EM, Nichlos CJ, Hickey KV, O'Rourke $\mathrm{P}$, et al. Laparoscopic sacral colpopexy versus total vaginal mesh for vaginal vault prolapse: a randomized trial. Am J Obstet Gynecol 2011; 204: 360.e1-7.

12. Maher C, Feiner B, Baessler K, Christmann-Schmid C, Haya N, Brown J. Surgery for women with apical vaginal prolapse. Cochrane Database Syst Rev 2016; 10: CD012376.

13. Maher C, Feiner B, Baessler K, Schmid C. Surgical management of pelvic organ prolapse in women. Cochrane Database Syst Rev 2013:CD004014.

14. Lowder JL, Park AJ, Ellison R, Ghetti C, Moalli P, Zyczynski H, et al. The role of apical vaginal support in the appearance of anterior and posterior vaginal prolapse. Obstet Gynecol 2008; 111: 152-7.

15. Eilber KS, Alperin M, Khan A, Wu N, Pashos CL, Clemens JQ, et al. Outcomes of vaginal prolapse surgery among female Medicare beneficiaries: the role of apical support. Obstet Gynecol 2013; 122: 981-7.

16. Rosati M, Bramante S, Bracale U, Pignata G, Azioni G. Efficacy of laparoscopic sacrocervicopexy for apical support of pelvic organ prolapse. JSLS 2013; 17: 235-44.

17. Aslam MF, Osmundsen B, Edwards SR, Matthews C, Gregory WT. Preoperative Prolapse Stage as Predictor of Failure of Sacrocolpopexy. Female Pelvic Med Reconstr Surg 2016; 22: 156-60.

18. Jackson SL, Weber AM, Hull TL, Mitchinson AR, Walters MD. Fecal incontinence in women with urinary incontinence and pelvic organ prolapse. Obstet Gynecol 1997; 89: 423-7.

19. Ellerkmann RM, Cundiff GW, Melick CF, Nihira MA, Leffler K, Bent AE. Correlation of symptoms with location and severity of pelvic organ prolapse. Am J Obstet Gynecol 2001; 185: 1332-7.

20. Jallad K, Gurland B. Multidisciplinary Approach to the Treatment of Concomitant Rectal and Vaginal Prolapse. Clin Colon Rectal Surg 2016; 29: 101-5. 
21. Campagna G, Panico G, Caramazza D, Anchora LP, Parello A, Gallucci V, et al. Laparoscopic sacrocolpopexy plus ventral rectopexy as combined treatment for multicompartment pelvic organ prolapse. Tech Coloproctol. 2020; 24: 573-84.

22. D'Hoore A, Cadoni R, Penninckx F. Long-term outcome of laparoscopic ventral rectopexy for total rectal prolapse. Br J Surg 2004; 91: 1500-5.

23. Randall J, Smyth E, McCarthy K, Dixon AR. Outcome of laparoscopic ventral mesh rectopexy for external rectal prolapse. Colorectal Dis 2014; 16: 914-9.

24. Emile SH, Elfeki H, Shalaby M, Sakr A, Sileri P, Wexner SD. Outcome of laparoscopic ventral mesh rectopexy for full-thickness external rectal prolapse: a systematic review, meta-analysis, and meta- regression analysis of the predictors for recurrence. Surg Endosc 2019; 33: 2444-55.

25. Persu C, Chapple CR, Cauni V, Gutue S, Geavlete P. Pelvic Organ Prolapse Quantification System (POP-Q) - a new era in pelvic prolapse staging. J Med Life 2011; 4: 75-81.

26. Brubaker L, Nygaard I, Richter HE, Visco A, Weber AM, Cundiff GW, et al. Two-year outcomes after sacrocolpopexy with and without burch to prevent stress urinary incontinence. Obstet Gynecol 2008; 112: 49-55.

27. Hudson CO, Northington GM, Lyles RH, Karp DR. Outcomes of robotic sacrocolpopexy: a systematic review and meta-analysis. Female Pelvic Med Reconstr Surg 2014; 20: 252-60. 